\title{
Bioactive starch-based scaffolds and human adipose stem cells are a good combination for bone tissue engineering
}

\author{
A.I. Rodrigues, M.E. Gomes, I.B. Leonor*, R.L. Reis* \\ 3B's Research Group - Biomaterials, Biodegradables and Biomimetics, Headquarters of the European Institute of Excellence on Tissue Engineering and Regenerative \\ Medicine, University of Minho, AvePark, 4806-909 Taipas, Guimarães, Portugal \\ ICVS/3B's - PT Government Associate Laboratory, Braga/Guimarães, Portugal
}

\section{A R T I C L E I N F O}

\section{Article history:}

Received 30 September 2011

Received in revised form 30 April 2012

Accepted 4 May 2012

Available online 29 May 2012

\section{Keywords:}

Bone tissue engineering

Human adipose stem cells

Silanol groups

Wet-spinning

Flow perfusion bioreactor

\begin{abstract}
A B S T R A C T
Silicon is known to have an influence on calcium phosphate deposition and on the differentiation of bone precursor cells. This study explores the effect of the incorporation of silanol ( $\mathrm{Si}-\mathrm{OH}$ ) groups into polymeric scaffolds on the osteogenic differentiation of human adipose stem cells (hASC) cultured under dynamic and static conditions. A blend of corn starch with polycaprolactone (30/70 wt.\%, SPCL) was used to produce three-dimensional fibre meshes scaffolds by the wet-spinning technique, and a calcium silicate solution was used as a non-solvent to develop an in situ functionalization with $\mathrm{Si}-\mathrm{OH}$ groups. In vitro assessment, using hASC, of functionalized and non-functionalized scaffolds was evaluated in either $\alpha$ MEM or osteogenic medium under static and dynamic conditions (provided by a flow perfusion bioreactor). The functionalized materials, SPCL-Si, exhibit the capacity to sustain cell proliferation and induce their differentiation into the osteogenic lineage. The formation of mineralization nodules was observed in cells cultured on the SPCL-Si materials. Culturing under dynamic conditions using a flow perfusion bioreactor was shown to enhance the hASC proliferation and differentiation and a better distribution of cells within the material. The present work demonstrates the potential of these functionalized materials for future applications in bone tissue engineering. Additionally, these results highlight the simplicity, economic and reliable production process of those materials.
\end{abstract}

(c) 2012 Acta Materialia Inc. Published by Elsevier Ltd. All rights reserved.

\section{Introduction}

Tissue engineering (TE) is a promising field for developing bone material capable of substituting for the conventional autogenic or allogenic transplants [1]. Currently, the general strategy for bone TE lies in biocompatible and biodegradable scaffolds seeded with stem cells [2]. However, those scaffolds have limited osteoconductivity and osteoinductivity, which compromise their use in bone TE [3]. Thus, the degree of success of bone TE greatly depends on the intrinsic properties of the material used to obtain a biocompatible and biodegradable scaffold with osteoinductive, osteoconductive and osteogenic properties in order to induce bone regeneration $[1,4,5]$.

Recently, silicate-based materials have been investigated, since they have the ability to activate bone-related gene expression and stimulate osteoblast proliferation and differentiation [6,7]. For

\footnotetext{
* Corresponding authors. Address: 3B's Research Group - Biomaterials, Biodegradables and Biomimetics, Headquarters of the European Institute of Excellence on Tissue Engineering and Regenerative Medicine, University of Minho, AvePark, 4806909 Taipas, Guimarães, Portugal. Tel.: +351 253510907.

E-mail addresses: belinha@dep.uminho.pt (I.B. Leonor), rgreis@dep.uminho.pt (R.L. Reis).
}

instance, bioactive ceramics, containing hydroxyapatite and silica, can degrade in proportion to the rate of new bone formation [8]. Thus, attention is focused on the importance of the chemical composition of materials, particularly the presence of silicon as a key player in enhancing bone repair. Moreover, culturing cells under dynamic conditions has been revealed to provide an important stimulus for the proliferation and differentiation of cells and, moreover, to mimic in vivo pressure gradients. Previous studies [9-12] showed that the use of a flow perfusion bioreactor enhances calcium deposition and osteogenic differentiation, and improves the cell distribution in a three-dimensional (3-D) scaffold.

In this context, the present authors aim to develop a biodegradable material with osteo stimulative properties combined with human adipose stem cells (hASC), which in future can serve as a platform for the development of bone implants able to replace the "gold standard" autograft [13,14].

In order to target this ambitious goal, a 3-D scaffold of SPCL (a blend of starch with polycaprolactone) was synthesized, incorporating $\mathrm{Si}-\mathrm{OH}$ groups by wet-spinning, using a calcium silicate solution as a coagulation bath, as described previously $[15,16]$. SPCL without $\mathrm{Si}-\mathrm{OH}$ groups, using methanol as a coagulation bath, was used as control. In vitro studies were performed by seeding and culturing wet-spun fibre mesh scaffolds, both with and 
without Si-OH groups, with hASC, either in a flow perfusion bioreactor or under static conditions. The physicochemical properties and biological response of hASC to the scaffolds developed were evaluated. It was hypothesized that this unconventional architecture, combined with the release of silicon (Si) into medium, would render an osteoconductive surface to the cells to which they would adhere, differentiate and produce a mineralized matrix, and in an in vivo situation would have an osteoinductive effect to induce bone regeneration.

\section{Materials and methods}

\subsection{Preparation of a bioactive polymeric fibre mesh by wet-spinning}

A biodegradable thermoplastic blend of corn starch with polycaprolactone (30/70 wt.\%, SPCL) was obtained from Novamont, Italy. Chloroform $\left(\mathrm{CHCl}_{3}\right)$, methanol $\left(\mathrm{CH}_{3} \mathrm{OH}\right)$, tetraethoxysilane (TEOS:$\left.\mathrm{Si}\left(\mathrm{OC}_{2} \mathrm{H}_{5}\right)_{4}\right)$ and calcium chloride $\left(\mathrm{CaCl}_{2}\right)$ were obtained from Sigma-Aldrich. Ethyl alcohol $\left(\mathrm{C}_{2} \mathrm{H}_{5} \mathrm{OH}\right)$ was obtained from Panreac.

To produce a bioactive 3-D structure, SPCL granules were dissolved in chloroform at a concentration of $30 \%(w / v)$ in order to obtain a polymeric solution with proper viscosity. Then, the polymeric solution was loaded into a $5 \mathrm{~mL}$ plastic syringe with a needle $(0.8 \mathrm{~mm}$ internal diameter) attached to it. The syringe was connected to a programmable syringe pump (KR analytical, precision syringe pump, Fusion 200; Chemics Inc., USA) to inject the polymeric solution at a controlled pump rate of $4.5 \mathrm{~mL} \mathrm{~h}^{-1}$. The fibre mesh structure was formed during the process by the random movement of the coagulation bath. Two different coagulation baths were used: (i) methanol, as control; and (ii) calcium silicate solution previously studied $[15,16]$. The calcium silicate solution was prepared by mixing TEOS, ultra-pure water, $\mathrm{C}_{2} \mathrm{H}_{5} \mathrm{OH}, 1.0 \mathrm{MHCl}$ aqueous solution and $\mathrm{CaCl}_{2}$ for $10 \mathrm{~min}$ in an ice bath with the following molar ratio $\mathrm{Si}\left(\mathrm{OC}_{2} \mathrm{H}_{5}\right)_{4} / \mathrm{H}_{2} \mathrm{O} / \mathrm{C}_{2} \mathrm{H}_{5} \mathrm{OH} /$ $\mathrm{HCl} / \mathrm{CaCl}_{2}$ of $1.0 / 4.0 / 4.0 / 0.014 / 0.20$. Using methanol, the formed fibre meshes were dried at room temperature overnight in order to remove any remaining solvent, and were designated as SPCL. In the case of using calcium silicate solution, the fibre meshes were dried in an oven at $60^{\circ} \mathrm{C}$ for $24 \mathrm{~h}$, and designated as SPCL-Si. Afterwards, two thin wet-spun fibre meshes were assembled to produce a 3-D bioactive scaffold with different properties in terms of porous size, interconnectivity, chemical characteristics and mechanical performance (Fig. 1).

Scaffolds were cut into $\varnothing 8 \mathrm{~mm}$ discs with thickness $\sim 1.16 \mathrm{~mm}$. Prior to any cell culture experiments, the scaffolds were sterilized by ethylene oxide, with a cycle time of $14 \mathrm{~h}$ at a working temperature of $45^{\circ} \mathrm{C}$ in a chamber under a pressure of $50 \mathrm{kPa}$. Six samples at a time were used for each experiment, separately for all time points and conditions.

\subsection{Characterization of wet-spun fibre mesh scaffolds}

For the morphological and the elemental characterization of the 3-D structures a field emission scanning electron microscope (NanoSEM, FEI Nova 200, USA) equipped with an energy dispersive spectroscope (Pegasus X4M) was used. Prior to any SEM observations, all scaffolds were coated with gold-palladium by ion sputtering. A graphite coating was used for energy dispersive spectroscopy (EDX) analysis to detect the possible presence of elements. The EDX analysis was performed using three samples for each condition.

The chemical structure of the surface of polymeric structures, SPCL and SPCL-Si, was analysed by Fourier-transformed IR spectroscopy with attenuated total reflectance (FTIR-ATR) in an IRPrestige-21 (Shimadzu, Japan). Spectra were collected at $4 \mathrm{~cm}^{-1}$ resolution using 60 scans in the spectral range 4400$800 \mathrm{~cm}^{-1}$. For each sample (SPCL and SPCL-Si), three individual measurements were performed.

The morphology and microstructure of fibre meshes scaffolds was evaluated using high-resolution micro-computed tomography $(\mu \mathrm{CT})$ with a Skyscan 1072 scanner (Skyscan, Kontich, Belgium). Xray scans were performed in triplicate, using a resolution of pixel size $4.17 \mu \mathrm{m}$ and integration time $2.1 \mathrm{~s}$. The X-ray source was set at energy $61 \mathrm{keV}$ and current $144 \mu \mathrm{A}$. Approximately 400 projections were acquired over a rotation range of $180^{\circ}$ with a rotation step of $0.45^{\circ}$. Data sets were reconstructed using standardized cone-beam reconstruction software (NRecon v1.4.3; SkyScan). Representative data sets of 40 slices were segmented into binary images with a dynamic threshold of 37-120 (grey values) to identify the organic and inorganic phases. These data were used for morphometric analysis (CT Analyser, v 1.5.1.5, SkyScan) and to build 3-D models (ANT 3D creator, v 2.4, SkyScan). The morphometric analysis included porosity. For this analysis, six samples were used.

The roughness of both scaffolds, SPCL and SPCL-Si, was characterized using a Wyko-NT 1100 interferometric optical profilometer (Veeco Instruments, USA) in vertical scanning interferometry (VSI) measurement mode. This is a non-contact optical profiling system that provides high resolution, 3-D surface measurement, from subnanometre surface roughness to millimetre step-height. The

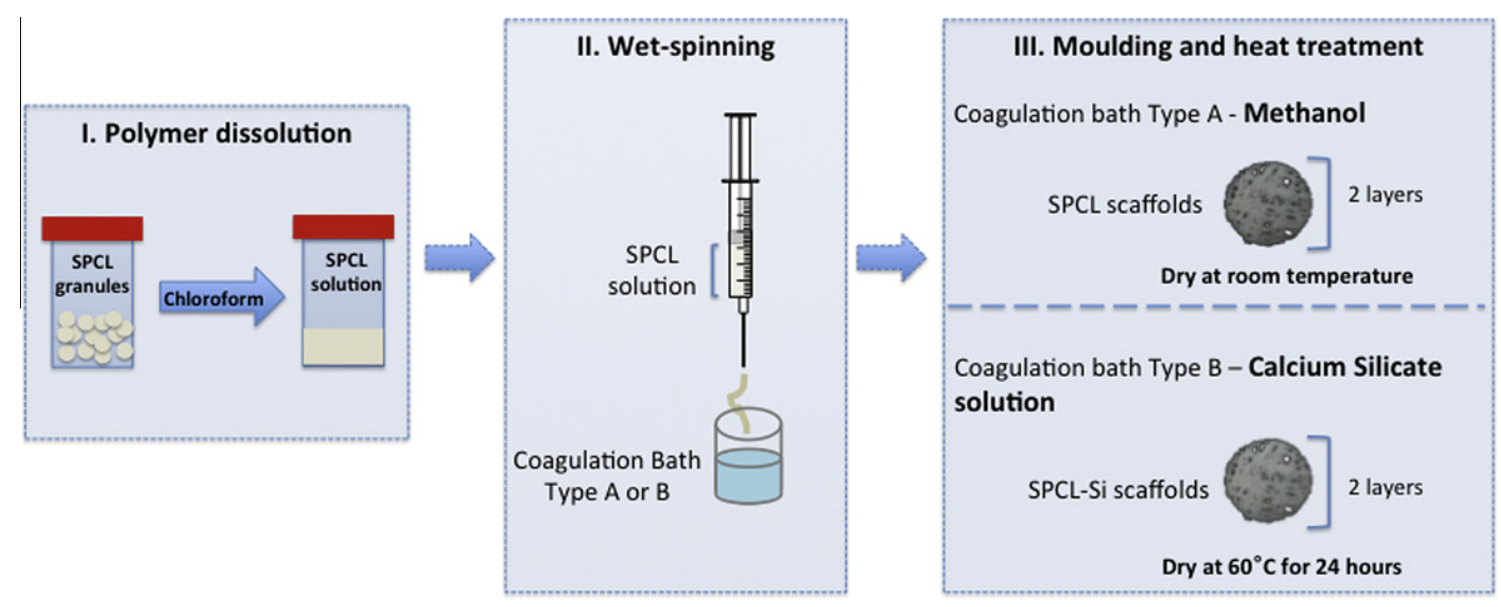

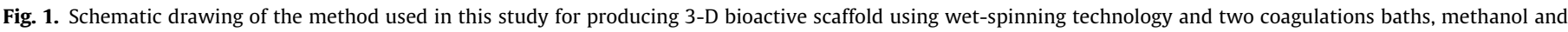
calcium silicate solution. A 3-D scaffold is made by stacking two layers of wet-spun fibre meshes. 
images were processed and analysed with the WycoVision ${ }^{\circledR} 32$ analytical software package. For each sample (SPCL and SPCL-Si), five individual measurements were performed.

\subsection{In vitro assessment of the wet-spun fibre mesh scaffolds}

\subsection{1. hASC harvesting and isolation}

hASC were harvested from two women 25 and 70 years old, under a protocol previously established with the Department of Plastic Surgery of "Hospital da Prelada" in Porto, Portugal, and isolated through an enzymatic procedure at different time points. ASC were enzymatically isolated from subcutaneous adipose tissue, as previously described [17]. The cells from each patient were used in two independent experiments. Briefly, the lipoaspirate samples were first washed with a solution of phosphate buffered saline (PBS) and $10 \%$ antibiotic/antimycoticsolution (A/B, Gibco) and then digested with $0.2 \%$ collagenase type II solution (Sigma) for $45 \mathrm{~min}$ with intermittent shaking, at $37^{\circ} \mathrm{C}$. Then the digested tissue was filtered using a $100 \mu \mathrm{m}$ filter mesh (Sigma-Aldrich), and the floating adipocytes were separated from the precipitated stromal vascular fraction by centrifugation at $1200 \mathrm{rpm}$ for $10 \mathrm{~min}$. Afterwards, the cell pellet was re-suspended in lysis buffer for $10 \mathrm{~min}$ to disrupt the erythrocytes and centrifuged at $800 \mathrm{rpm}$ for $10 \mathrm{~min}$. Finally, the cells were again re-suspended and placed in culture flasks with minimum essential $\alpha$-medium ( $\alpha$-MEM) (Gibco) supplemented with sodium bicarbonate, A/B and 10\% (v/ v) of fetal bovine serum (FBS) (Gibco) at $37{ }^{\circ} \mathrm{C}$ with $5 \% \mathrm{CO}_{2}$. Until the cells reached $80-90 \%$ confluence, the medium was changed every 2 days.

\subsubsection{Static and dynamic perfusion culturing of human ASC on fibre mesh scaffold}

For the in vitro tests, two independent experiments were performed, and in each experiment six samples were used per condition and culture time. Scaffolds without cells were used as controls for all studies.

Cells were expanded, sub-cultured twice (passage 2) and seeded onto SPCL and SPCL-Si scaffolds in 48-well plates at a density of $5 \times 10^{5}$ cells/scaffold in a $100 \mu \mathrm{L}$ cell suspension and incubated for $2 \mathrm{~h}$. Then, $900 \mu \mathrm{L}$ of $\alpha$-MEM was added to the wells containing cell-scaffolds constructs, and incubated $24 \mathrm{~h}$ to allow cell attachment. Afterwards, cell-scaffold constructs were either kept in the well cell culture plate (static culture) or transferred to a flow perfusion bioreactor (dynamic culture) and further incubated for 1, 7, 14 and 21 days. In static culture, the culture medium was changed every 3 days. In both cases, static and dynamic, the experiments were carried out using $\alpha$-MEM or osteogenic medium containing $\alpha$-MEM, 10\% FBS, $1 \%$ A/B and osteogenic supplements (ascorbic acid $\left(50 \mu \mathrm{g} \mathrm{ml}^{-1}\right)$, dexamethasone $\left(10^{-8} \mathrm{M}\right)$ and $\beta$-glycerophosphate $(10 \mathrm{mM}))$.

The flow perfusion bioreactor used for dynamic culture was developed in the 3B's research group [18] and is composed of a main chamber that can hold up to 20 samples of $\varnothing 8 \mathrm{~mm}$. The culture medium is pumped from a medium reservoir into the bioreactor chamber by a peristaltic pump (Ismatec), which allows a homogeneous distribution of the medium through scaffolds in the individual containers of the perfusion chamber. In this study, the flow rate was based on previous studies, and it was fixed to $0.1 \mathrm{~mL} \mathrm{~min}^{-1}$ per scaffold $[9,11,19]$. The total volume of medium in the flow system was $100 \mathrm{~mL}$ and was renewed every week. The flow perfusion bioreactor was maintained in $5 \% \mathrm{CO}_{2}$ and incubated at $37^{\circ} \mathrm{C}$ for up to 21 days.

\subsubsection{Cell morphology evaluation}

The morphology of hASC cultured onto the fibre meshes was analysed by SEM. The cell-scaffold constructs were washed with
PBS, fixed with a $2.5 \%$ gluteraldehyde solution (Sigma) for $1 \mathrm{~h}$ at $4{ }^{\circ} \mathrm{C}$. Then, the samples were dehydrated in a series of ethanolwater solutions with increasing ethanol concentration (30\%, 50\%, $70 \%, 90 \%$ and $100 \%, v / v$ ) and treated with hexamethyldisilazane. Afterwards, the samples were left to dry overnight.

\subsubsection{Cell attachment, proliferation and differentiation}

To investigate the attachment, proliferation and differentiation of hASC, the cell-scaffold constructs were removed after 1, 7, 14 and 21 days, rinsed with PBS and transferred into eppendorf containing sterile ultrapure water. Then, the samples were frozen at $-80^{\circ} \mathrm{C}$ and defrosted at room temperature before the DNA and alkaline phosphatase (ALP) assays. Triplicates were analysed for each sample at each time point for both assays.

2.3.4.1. DNA assay. The DNA content in the scaffolds was determined using the fluorescent picoGreen dsDNA (ds, double stranded) quantification assay (Invitrogen Corporation, USA). Samples previously frozen $\left(-80^{\circ} \mathrm{C}\right)$ were thawed at room temperature and then sonicated for $15 \mathrm{~min}$ to induce complete membrane lysis. Supernatant fluorescence was measured $(485 \mathrm{~nm}$ excitation and $528 \mathrm{~nm}$ emission) in a microplate reader (Synergy HT, BioTek Instruments, USA) and the DNA amounts calculated according to a standard curve.

2.3.4.2. ALP assay. ALP activity from cell-scaffold constructs $(n=6)$ was quantified by the specific conversion of $p$-nitrophenyl phosphate (pNPP, Sigma) to $p$-nitrophenol (pNP, Sigma). A buffer solution containing $0.2 \%(\mathrm{w} / \mathrm{v}) \mathrm{pNPP}$ was added to the supernatant in a 96-well plate (Costar, Becton Dickinson). The enzyme reaction was carried out at $37{ }^{\circ} \mathrm{C}$ for $45 \mathrm{~min}$ and then stopped by a solution containing $2 \mathrm{M} \mathrm{NaOH}$ and $0.2 \mathrm{mM}$ EDTA in distilled water. The absorbance of pNP formed was read at $405 \mathrm{~nm}$ in a microplate reader (Synergy HT, BioTek Instruments, USA). A standard curve was made using pNP values ranging from 0 to $0.2 \mathrm{mmol} \mathrm{ml}^{-1}$.

\subsection{Statistical analysis}

All the quantitative results were obtained from triplicate samples. Data are reported as mean \pm standard deviation. For statistical analysis, a one-way ANOVA test was performed, and the differences were considered statistically significant if $p<0.05$.

\section{Results}

The functionalization of the starch-based materials was achieved by combining wet-spinning with a calcium silicate solution as a coagulation bath in one-step approach. Different parameters were used in order to optimize the production of those materials for biological assays. This work was performed to evaluate the incorporation of $\mathrm{Si}-\mathrm{OH}$ groups into the fibre meshes on the adhesion, differentiation and proliferation of hASC when cultured under dynamic conditions.

\subsection{Wet-spun fibre mesh scaffolds characterization}

The wet-spun fibre mesh scaffolds were observed using a magnifying glass, SEM and EDX elemental mapping of carbon (C), oxygen (O), calcium (Ca) and silicon (Si) (Fig. 2).

From the images obtained from the magnifying glass and SEM, one can see a uniform distribution of the fibres in both types of scaffolds. Also, from SEM observation, the SPCL-Si material presents a smooth surface compared with SPCL. This assumption was supported by optical profilometer analysis to evaluate the eventual topographical changes on the surface of the fibres as a 



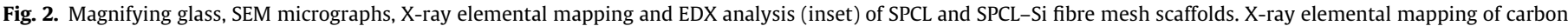
$(\mathrm{C}$, red), oxygen ( $\mathrm{O}$, green), silicon ( $\mathrm{Si}$, blue) and calcium (Ca, yellow).

result of using two different coagulation baths, methanol and calcium silicate solution. It is shown in the optical profile images (Supporting Information, Fig. S1) that no significant topography changes were detected, meaning that SPCL and SPCL-Si samples show very similar topography. The calculated value of average roughness $(\mathrm{Ra})$ from $100 \times$ magnification images of SCPL sample was $\mathrm{Ra}=177.58 \pm 22.63 \mathrm{~nm}$, which was slightly higher than that obtained for the SPCL-Si sample, Ra $=164.70 \pm 18.07 \mathrm{~nm}$.

$\mu C T$ analysis indicated that SPCL and SPCL-Si scaffolds have a typical fibre mesh structure, with a fibre diameter $\sim 121.94 \pm$ $24.9 \mu \mathrm{m}$ and $117.5 \pm 13.04 \mu \mathrm{m}$, respectively. In terms of porosity, SPCL fibre meshes presented $84.3 \%$ and SPCL-Si fibre meshes $\sim 56 \%$, where the pores are interconnected (data not shown). Also, $\mathrm{X}$-ray elemental mapping of $\mathrm{Si}$ and $\mathrm{Ca}$ indicated a uniform distribution of those elements on the surface of SPCL-Si fibre meshes when using the calcium silicate solution (Fig. 2). EDX spectrum showed the presence of $\mathrm{Ca}$ and $\mathrm{Si}$ elements in the SPCL-Si scaffolds, although in the case of SCPL scaffolds those elements were not detected (inset in the X-ray elemental mapping in the Fig. 2).

FTIR-ATR characterization was used to study the chemical interaction between the calcium silicate solution and SPCL solution. Fig. 3 shows an absorption peak at $\sim 1725 \mathrm{~cm}^{-1}$ for both materials, which is attributed to the $\mathrm{C}=\mathrm{O}$ absorption band of $\mathrm{PCL}$, but a decrease is observed for the SPCL-Si. In the case of SPCL-Si fibre meshes, reflection

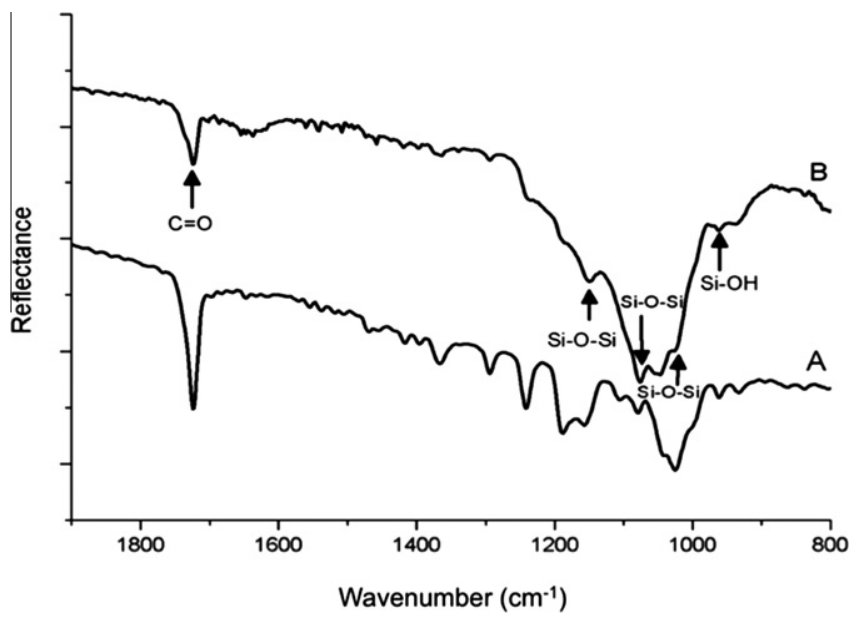

Fig. 3. FTIR-ATR spectrum of (A) SPCL and (B) SPCL-Si fibre meshes scaffolds. peaks at $\sim 1020$ and $1090 \mathrm{~cm}^{-1}$ and a shoulder at $\sim 1190 \mathrm{~cm}^{-1}$ were observed and can be related to siloxane bonds ( $\mathrm{Si}-\mathrm{O}-\mathrm{Si}$ ). At $\sim 970 \mathrm{~cm}^{-1}$ corresponds to $\mathrm{Si}-\mathrm{OH}$, typically observed in a silica gel.

\subsection{In vitro assessment of wet-spun fibre mesh scaffolds}

\subsection{1. hASC morphology}

SEM was used to examine the adhesion and morphology of hASC cultivated on the fibre meshes. Fig. 4 presents SEM micrographs of hASC on SPCL and SPCL-Si materials after 1, 7, 14 and 21 days of culture in $\alpha$-MEM and osteogenic medium under static and dynamic conditions.

The presence of $\mathrm{Si}-\mathrm{OH}$ groups induces a significant difference in the distribution of hASC cultured in either $\alpha$-MEM or osteogenic medium under static and dynamic conditions.

Using $\alpha$-MEM, the cells revealed lower adhesion and proliferation levels than cells cultured in osteogenic medium, in both materials. Cells cultured on SPCL-Si scaffolds, under all conditions, exhibited a flat and elongated shape, as observed in Fig. 4. The cells can be seen stretching and forming bridges from one fibre to another on the scaffolds. Also, hASC were observed to spread within the pores of the SPCL-Si fibre meshes. After 14 days in osteogenic medium/dynamic culture, the hASC cultured on SPCL-Si formed a dense cell layer covering the surface, whereas those cultured on $\alpha$-MEM formed only a monolayer that partially covers the surfaces. After 21 days in $\alpha$-MEM and osteogenic medium under dynamic conditions, the SPCL-Si fibre mesh scaffolds were completely covered by a cell layer, while cells cultured on SPCL scaffolds presented only some filopodia between the fibres. Also, on SPCL scaffolds it was possible to observe that some cells still present a spindle-like shape, while others are starting to elongate. After 14 and 21 days, one can also observe that the cells have already penetrated the interior of the scaffolds and, on SPCL-Si scaffolds, the cells started to stretch and form bridges between the fibres. And once more, for SPCL scaffolds it was not possible to observe the formation of dense cell layer in any of the conditions studied.

\subsection{2. hASC proliferation}

hASC proliferation was assessed by quantifying cellular DNA, as shown in Fig. 5.

A significantly higher $(p<0.05)$ cell proliferation was observed in the case of SPCL-Si constructs compared with cell-seeded SPCL scaffolds, which is supported by the proliferation factors where, 

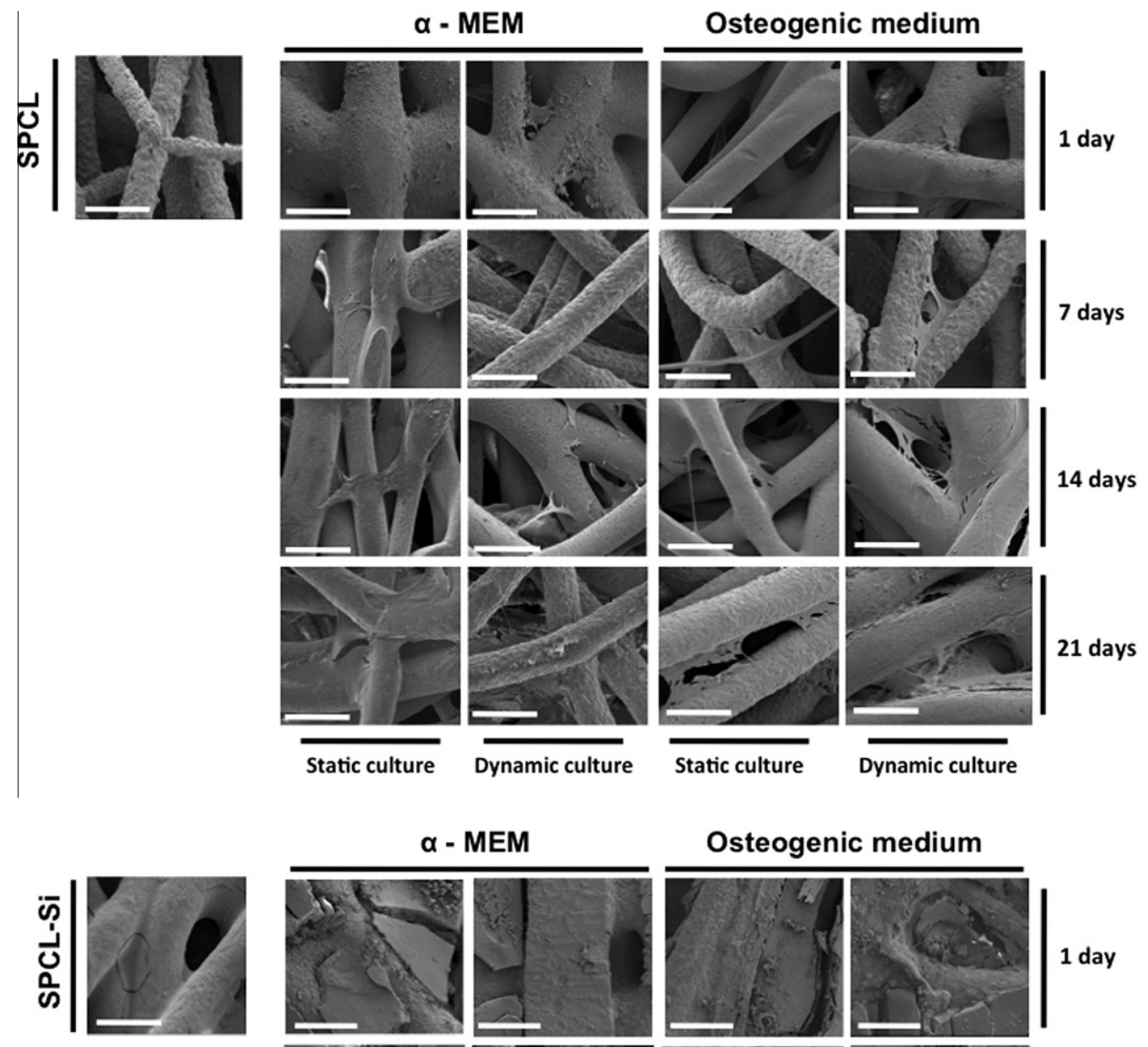

\section{Osteogenic medium}
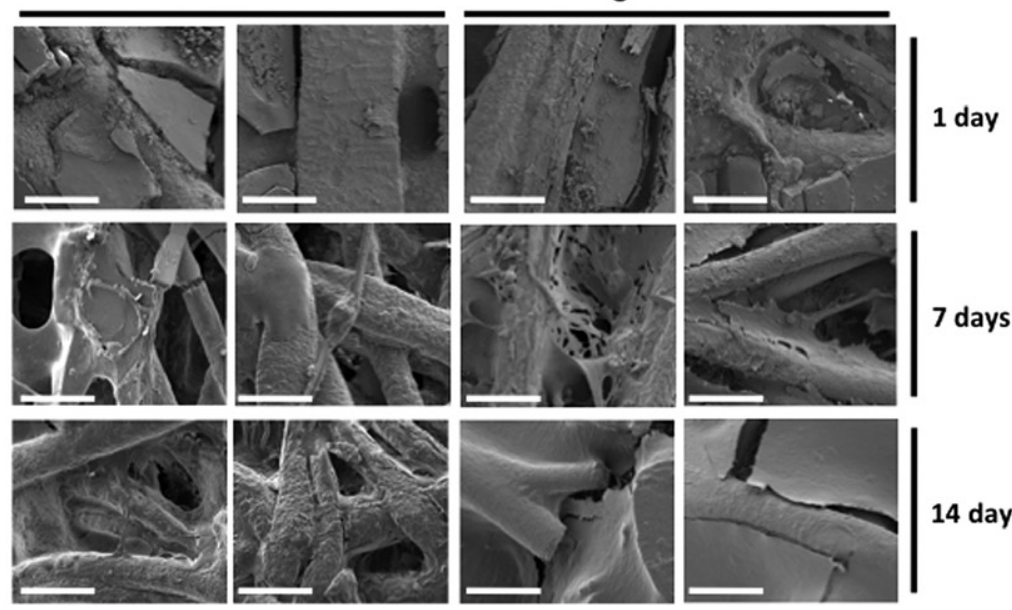

14 days

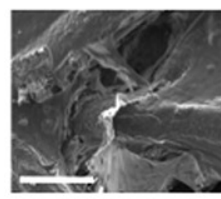

Static culture

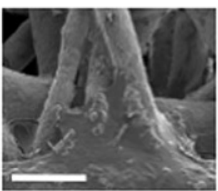

$\overline{\text { Dynamic culture }}$

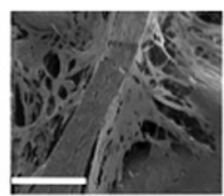

Static culture

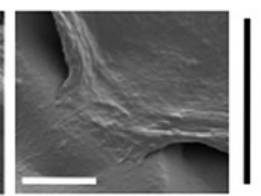

21 days

$\overline{\text { Dynamic culture }}$

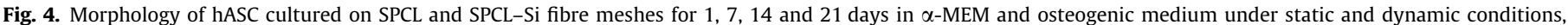
observed by SEM. In all assays, SPCL and SPCL-Si fibre meshes without cells were used as controls. Scale bar corresponds to $200 \mu \mathrm{m}$.

at 7 days for $\alpha$-MEM under dynamic conditions, the values were 6.4 for SPCL-Si and 0.7 for SPCL.

Results from day 1 indicate a low initial cell attachment. However, the proliferation factors indicate a high proliferation from day 1 to day 7 , especially under dynamic conditions using osteogenic medium, which compensates the initial lower cell attachment: 5.9 for SPCL and 7.9 for SPCL-Si, respectively. Concerning the influence of culture conditions, the DNA quantification values of the cells cultured under dynamic conditions are higher than those cultured under static conditions.

\subsection{3. hASC differentiation}

The ALP activity of hASC was assessed as an indicator of the osteogenic differentiation. Fig. 6 shows the normalized ALP expression by hASC on the scaffolds up to 21 days.

The results obtained showed a higher ALP activity under dynamic culture conditions in osteogenic medium, both for SPCL and SPCL-Si fibre meshes, compared with static culture conditions in $\alpha$-MEM. However, in the case of SPCL-Si constructs, the ALP values were lower than on SPCL constructs for all the conditions. 


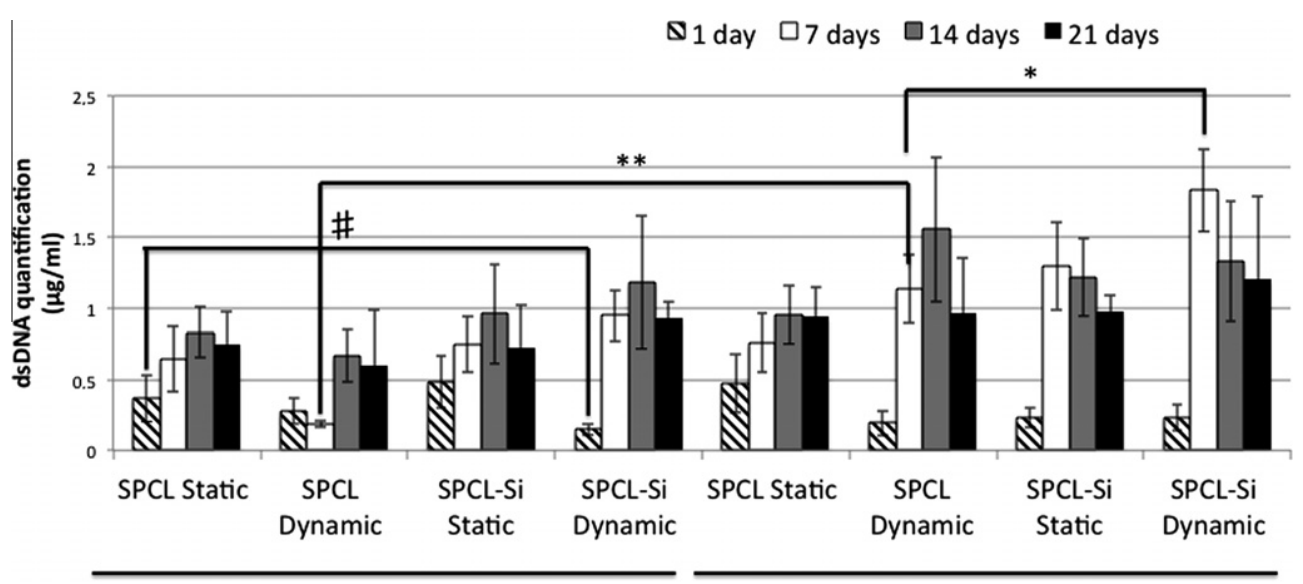

$\alpha-$ MEM

Osteogenic medium

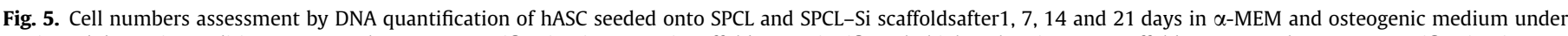

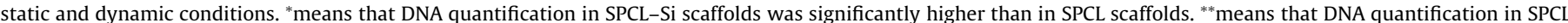

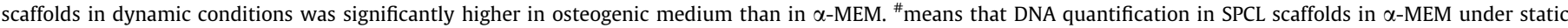
conditions was significantly higher than in SPCL-Si scaffolds in $\alpha$-MEM under dynamic conditions. $(p<0.05 ; n=6)$.

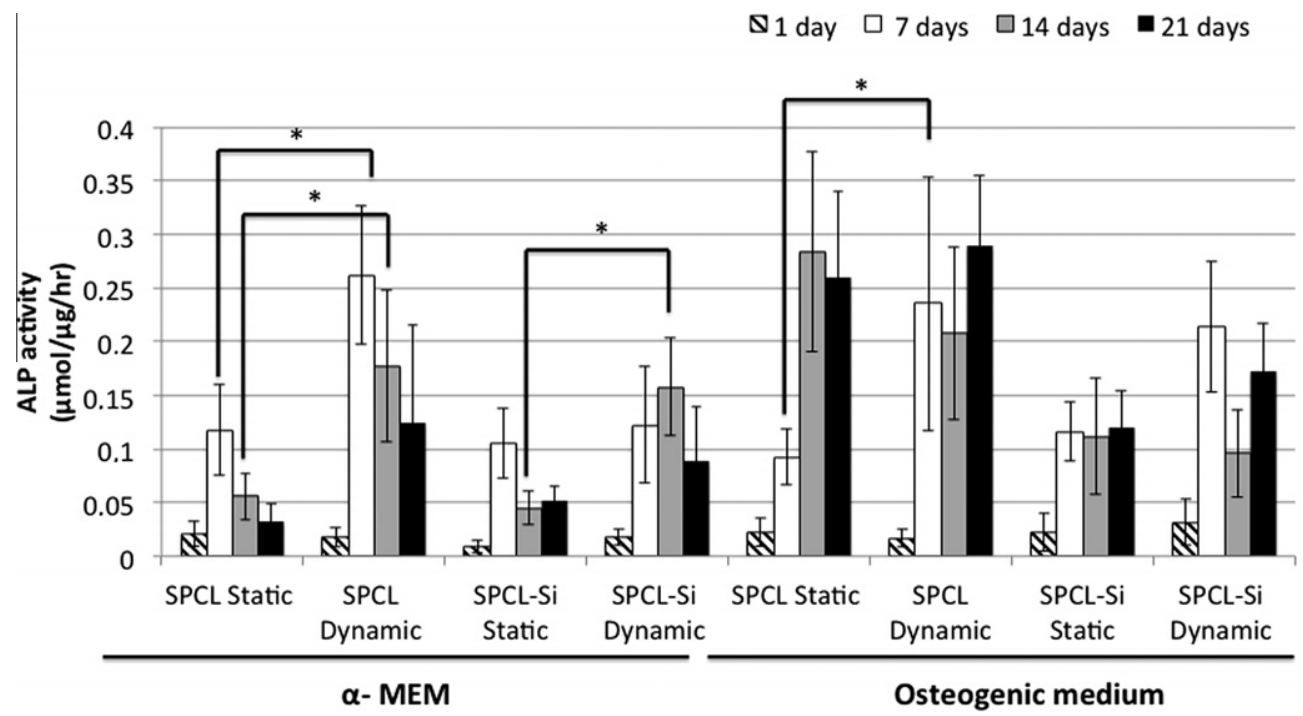

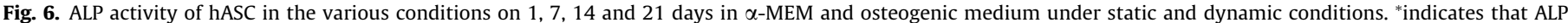
activity was significantly higher $(p<0.05)$ in cells cultured under dynamic conditions than under static conditions. $(p<0.05$; $n=6)$. Values were normalized.

FTIR-ATR analysis performed on the SPCL and SPCL-Si fibre meshes cultured with cells for 14 days under different conditions revealed the presence of several groups, as shown in the Fig. 7 .

The amide I, II and III bands were detected at $\sim 1635 \mathrm{~cm}^{-1}$, $\sim 1532 \mathrm{~cm}^{-1}$ and $\sim 1200 \mathrm{~cm}^{-1}$ for SPCL and SPCL-Si fibre meshes for all conditions, however the intensity of those bands differs between the materials. The presence of $\mathrm{PO}_{4}^{3-}$ groups was detected at $\sim 1078 \mathrm{~cm}^{-1}$ for SPCL-Si scaffolds for all conditions, although their intensity differs. For SPCL materials, a phosphate band $\sim$ at $1090 \mathrm{~cm}^{-1}$ was also detected. However, the intensity of this band in the SPCL materials was lower compared with the SPCL-Si scaffolds cultured in $\alpha$-MEM.

Regarding the hASC differentiation, mineralization nodules were observed in SPCL-Si constructs by SEM after 14 days in osteogenic medium under static conditions (Fig. 8). The EDX analysis confirmed that nodules were composed mainly of Ca and P. Also, in the case of SPCL-Si cultured in osteogenic medium under dynamic conditions, the presence of $\mathrm{Ca}$ and $\mathrm{P}$ was detected, but their intensities were lower. In the SPCL scaffolds, it was not possible to observe the presence of mineralization nodules; neither $\mathrm{P}$ nor $\mathrm{Ca}$ elements were detected (Fig. 8). These results are in agreement with the $\mathrm{Ca} / \mathrm{P}$ ratios obtained by EDX analysis. For SPCL-Si constructs cultured in $\alpha$-MEM under static and dynamic conditions, the $\mathrm{Ca} / \mathrm{P}$ values were $\sim 1.53$ at day 14 (Fig. 9). The $\mathrm{Ca} / \mathrm{P}$ ratios for the SPCL constructs cannot be calculated, as the $\mathrm{Ca}$ and $\mathrm{P}$ ions were not detected by EDX analysis.

In osteogenic medium under dynamic conditions, the $\mathrm{Ca} / \mathrm{P}$ ratio reached higher values between 2.9 and 3.1. An increase in the $\mathrm{Ca} / \mathrm{P}$ ratio as function of time was also observed in the osteogenic medium. For 1 day of culture, the presence of P on the surface of SPCL$\mathrm{Si}$ was not detected, only $\mathrm{Ca}$, and therefore the values of $\mathrm{Ca} / \mathrm{P}$ ratio were not included. For the controls, without cells, only the presence of $\mathrm{P}$ was detected, which can be due to the presence of this element in both media, osteogenic and $\alpha$-MEM, but the values were very low. Concerning the SPCL materials cultured with hASC, since EDX analysis did not detect the presence of phosphorous, the $\mathrm{Ca} / \mathrm{P}$ ratio was not calculated.

The measurement of the release of $\mathrm{Si}$ into the medium along the culture time gives a possible explanation for hASC osteogenic differentiation. Thus, the presence of Si in both samples along the cul- 

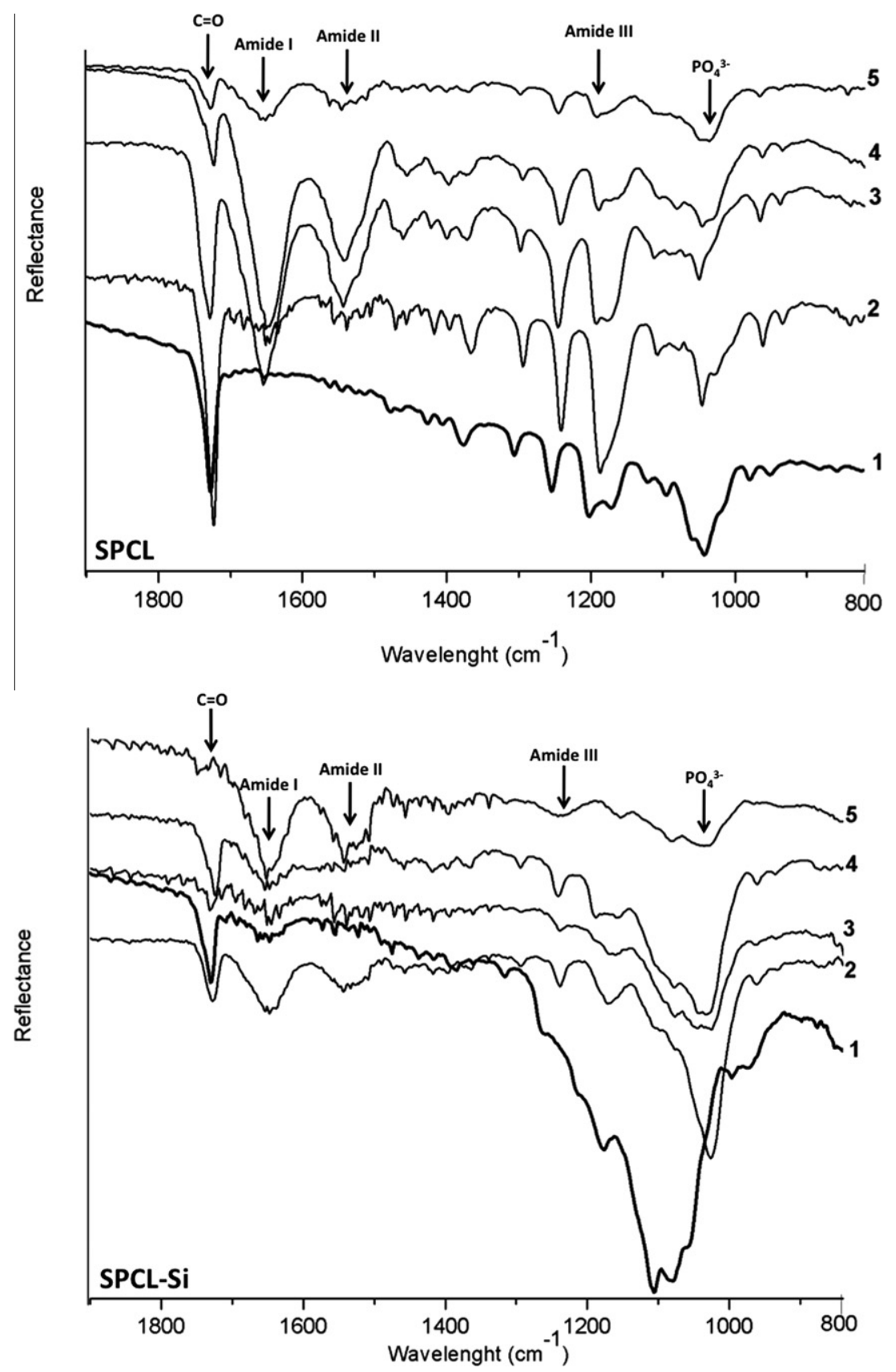

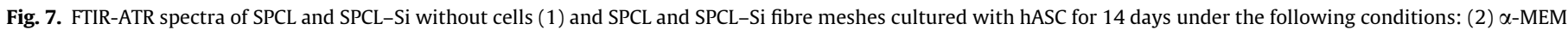
medium/static condition; (3) osteogenic medium/static condition; (4) $\alpha$-MEM medium/dynamic condition; (5) osteogenic medium/dynamic condition.

ture time was quantified by EDX analysis and indirectly determines its release to the culture medium. In Fig. 10, a clear decrease in $\mathrm{Si}$ (especially during the initial period) was observed in the material as a function of time, demonstrating its release to the culture medium. The decrease in Si on the surface of the scaffolds cultured under static conditions was lower than in those cultured under dynamic conditions. Also, at day 1 and 7, one can observe that the release of $\mathrm{Si}$ from materials cultured on basal medium was lower.

\section{Discussion}

Using wet-spinning methodology, one is able to produce fibre meshes at lower temperatures, which can enable the incorporation of biological molecules. In previous work [16], it was demonstrated that it was feasible to produce bioactive 3-D fibre meshes with $\mathrm{Si}-$ $\mathrm{OH}$ functional groups on their surface, with a controlled pore size and orientation, in a one-step procedure in a reliable and economical way. This simple process has the advantage that no further coating or chemical modification of the fibre mesh is required to render bioactive behaviour as in classic ceramic materials, such as an organized arrangement of functional groups, silanol ( $\mathrm{Si}-$ $\mathrm{OH})$ groups.

Recent studies [20-23] suggest that silicon is an essential mineral that promotes and maintains osteogenesis, since it is required for the growth and production of mucopolysaccharides and collagen in connective tissues. Moreover, silicon and calcium ions, act on the skeletal system during its growth and development by regulating and activating genes in osteoprogenitor cells. Thus, the 

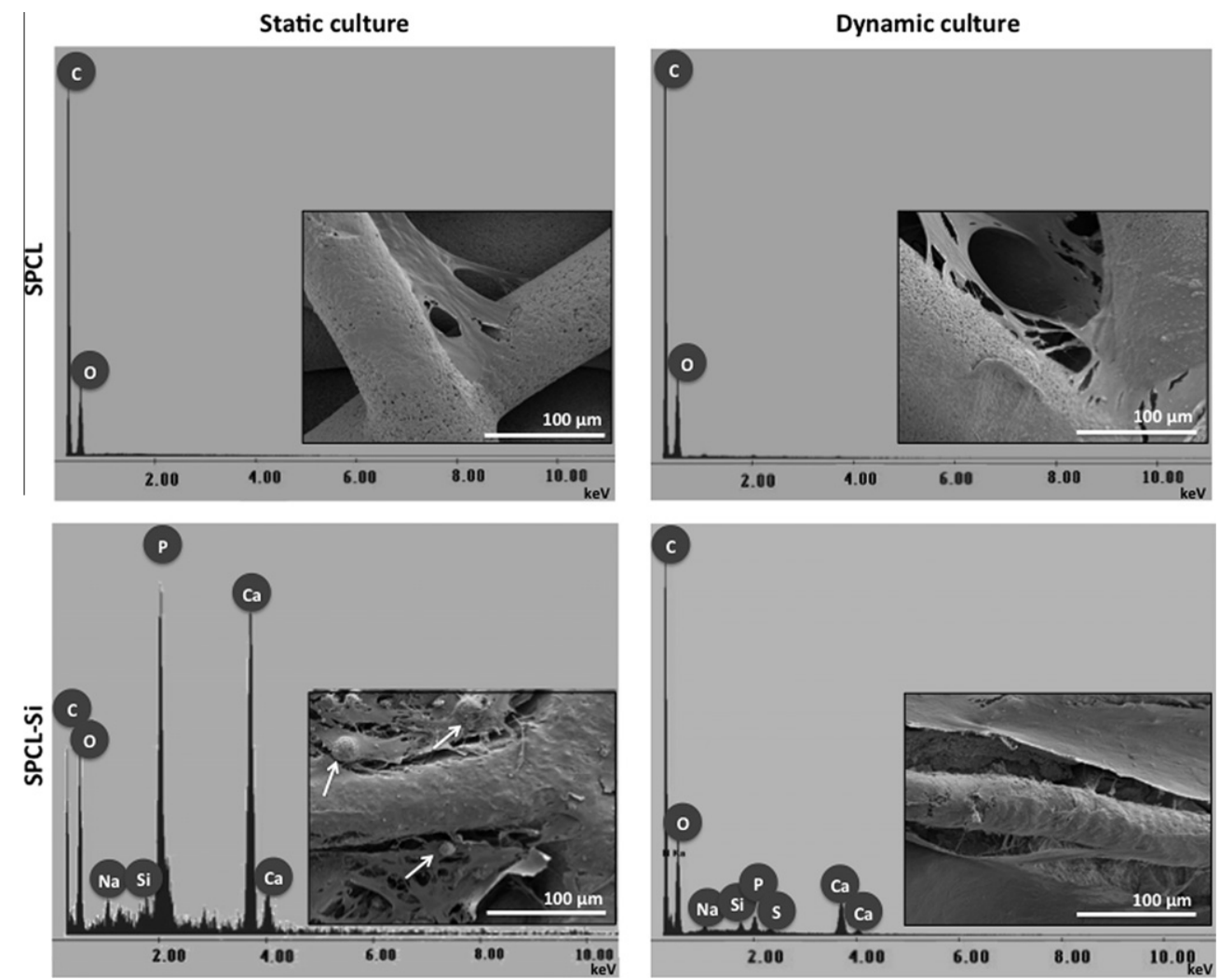

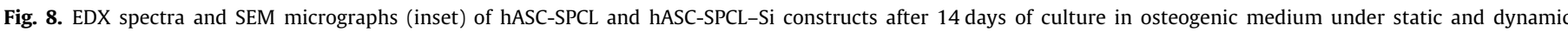
conditions. The white arrows indicate mineralization nodules.

main aim of this work is to improve the in vitro osteogenic functionality of cell-scaffold constructs, incorporating $\mathrm{Si}-\mathrm{OH}$ groups, by stimulating the osteogenic and/or matrix production using a flow perfusion bioreactor. It was expected with this approach to develop a bioactive scaffold able to promote bone regeneration.

Before the in vitro assessment, the wet-spun fibre mesh scaffolds were analysed by X-ray elemental mapping in order to observe the distribution of $\mathrm{Si}$ and $\mathrm{Ca}$ on the materials (Fig. 2). The results obtained show a uniform distribution of the elements on the surface of functionalized materials, SPCL-Si. Also, the presence of P and Ca elements in SPCL-Si scaffolds was detected by EDX analysis (inset in the X-ray elemental mapping in the Fig. 2), which is in agreement with the data obtained from X-ray elemental mapping. These results indicate that, using a calcium silicate solution as the coagulation bath, it is possible to functionalize 3-D structures by wet-spinning.

The wet-spun fibre mesh scaffolds were also characterized by FTIR-ATR analysis to evaluate the interactions between the organic and inorganic phases after the precipitation of SPCL polymeric solution into the coagulation bath, calcium silicate solution. Fig. 3 shows that the components typical of PCL at $1725 \mathrm{~cm}^{-1}$ $(\mathrm{C}=\mathrm{O})$ and $1170 \mathrm{~cm}^{-1}(\mathrm{C}-\mathrm{O})$ decrease their intensity, while a new shoulder band appeared at $1210 \mathrm{~cm}^{-1}[15,24]$. The decrease in stretching vibrations of carbonyl and ether groups could be related to the incorporation of silicon from the precipitation of SPCL polymeric solution in the coagulation bath calcium silicate solution. Also, several characteristic bands of sol-gel-derived silica were observed. The presence of $\mathrm{Si}-\mathrm{OH}$ groups comes from the hydrolysed and incomplete polycondensation reaction of TEOS, which can lead to the formation of hydrogen-bonding interactions with carbonyls of PCL. For instance, the presence of a new shoulder could be attributed to the stretching vibration of carbonyl groups of PCL that could be bonded with the silanols groups issuing from the calcium silicate solution. Several research works have demonstrated these hydrogen-bonding interactions between carbonyls of PCL and $\mathrm{Si}-\mathrm{OH}$ of silica network in the hybrid materials [25-27]. In these works, the inorganic-organic hybrids are prepared via an in situsol-gel process of TEOS in the presence of PCL. In a previous work [15], X-ray photoelectron spectroscopy was performed, and those results are in accordance with the FTIR-ATR data presented here, confirming the incorporation of $\mathrm{Si}-\mathrm{OH}$ groups.

It is well known that the surface characteristics of a biomaterial play a key role in modulating osteoblast adhesion, influencing cell morphology and its proliferation and differentiation. Owing to the importance of this interface, biomaterial surface and stem cells, more research has been done trying to tailor 3-D structures with chemical and biological signals for promoting a specific cellular phenotype, instead of using biocompatible and biodegradable scaffolds seeded with appropriate stem cells, the common strategy used in bone TE $[28,29]$. Looking at the SEM micrographs, the differences in the surface between the SCPL-Si and SPCL scaffolds are clear, and therefore it is important to clarify whether those differences have an impact on cell attachment and proliferation. For that, the roughness of both materials was measured, where the roughness of SPCL was slightly higher than that obtained for SPCL-Si samples. However, the values in both samples were in the same order of magnitude, suggesting that using a calcium silicate solution as a coagulation bath did not produce significant changes in topography as shown by optical profilometry (Supporting Information, Fig. S1). Thus, the results obtained suggest that it is the surface chemistry of the scaffolds and not the roughness that influences cell differentiation.

In general, the outcomes from the in vitro assessment of SPCLSi wet-spun fibre mesh scaffolds are better than those reported for the control, non-functionalized scaffolds. However, the results obtained demonstrate that, besides the scaffold composition, the 


\section{$\alpha-$ MEM}

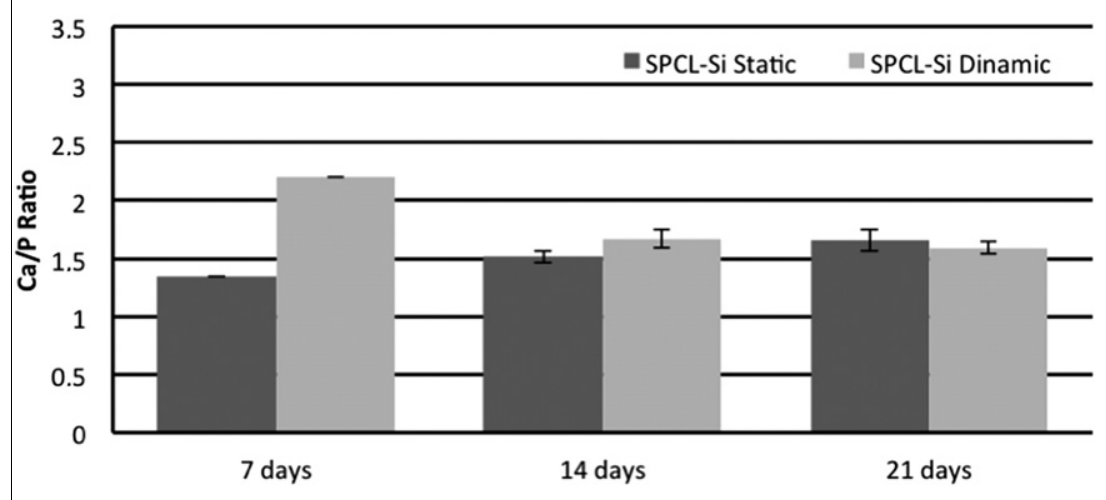

Osteogenic medium

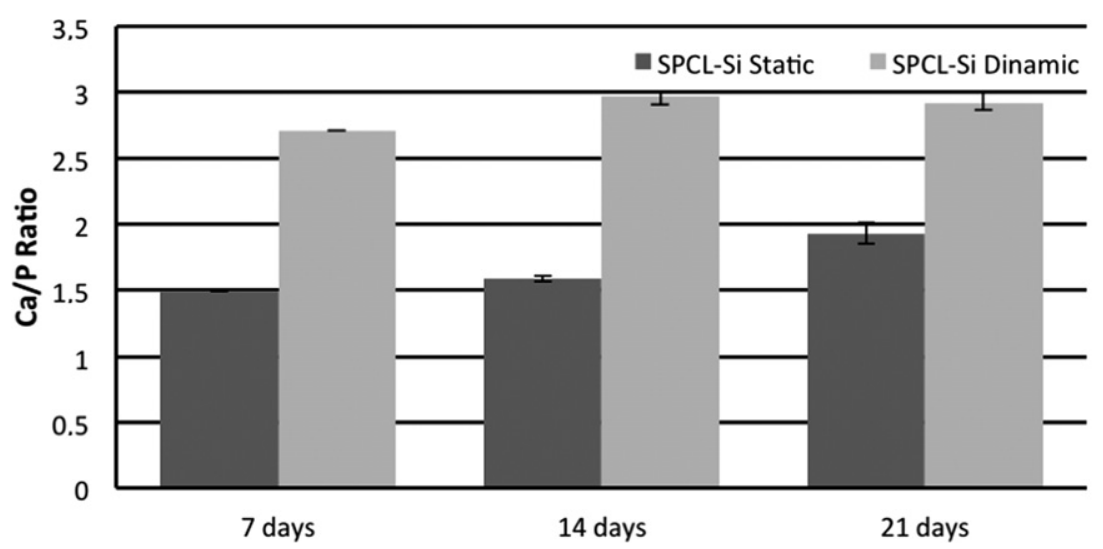

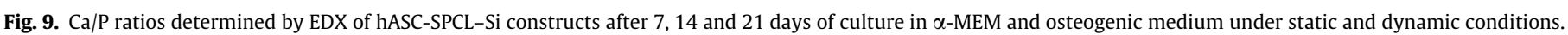
Data are shown as mean \pm standard deviation from $n=3$ samples.

culture conditions (static and dynamic) and culture medium ( $\alpha$ MEM and osteogenic medium) produce significant differences in the cell behaviour.

The morphology of hASC observed by SEM showed that cells on SPCL-Si scaffolds, under all conditions, exhibited a flat and elongated shape, typical of mature osteoblasts [30]. In the dynamic culture, the cells penetrated the SPCL-Si scaffolds and formed a dense matrix, which demonstrates that the constant fluid flow provides an adequate distribution of nutrients and better removal of metabolic waste. All of these are fundamental for higher cell proliferation, as previously demonstrated by other studies $[9,11,12]$. Also, the formation of filopodia into the pores of the SPCL-Si scaffolds is in agreement with the results of the study by Haimi et al. [31], where the hASC are combined with bioactive glass-based scaffolds. For SPCL scaffolds, some cells presented a spindle-like morphology typical of hASC [30], while other cells were starting to elongate and to acquire a flat shape.

Cell proliferation results by DNA quantification indicate that SPCL-Si fibre meshes with adequate porosity are able to support and sustain hASC proliferation under dynamic conditions using osteogenic medium (Fig. 5). These results can be explained by the release of Si to the culture medium. Furthermore, the presence of osteogenic supplements, namely ascorbic acid, dexamethasone and $\beta$-glycerophosphate, in the culture medium can also have a certain influence in those results. Previous studies [21,32,33] have shown increased proliferative activity in materials containing Si. Concerning the influence of osteogenic factors in the medium, especially ascorbic acid, it has been demonstrated that they act as positive modulators of hASC proliferation [34].

Cells cultured using the flow perfusion bioreactor revealed increased proliferation, which can be explained by a better distribution of oxygen and nutrients due to the constant flow and the mechanical stimuli provided by the bioreactor. This increase has been shown in previous studies, using a flow perfusion bioreactor [9,11,12]. The same behaviour was observed by Fröhlich et al. [35] using hASC and a perfusion bioreactor, which supports the present results. The higher value of DNA content in SPCL samples cultured on osteogenic medium/dynamic conditions, namely at day 7 , showed a significant higher cell number compared with the SPCL samples cultured on basal medium/dynamic conditions (Fig. 5). This can be related to different stages of differentiation of hASC when cultured under dynamic conditions, since the bioreactor provides a mechanical stimulation to cells via shear stress induced by the medium flow. During the last 2 weeks of culture, under static conditions using osteogenic medium the proliferation decreased for SCPL-Si scaffolds, which is supported by the proliferation factors. Also, these results are in agreement with the observation by SEM showing the formation of mineralized nodules, and with the analysis by EDX confirming the presence of $\mathrm{P}$ and $\mathrm{Ca}$ (Fig. 8). These results are in agreement with the fact that, when the cells start to differentiate, their proliferation rate decreases [36].

The evaluation of cell differentiation and mineralization was characterized using different analytical techniques, namely, ALP activity, FTIR-ATR and EDX. 


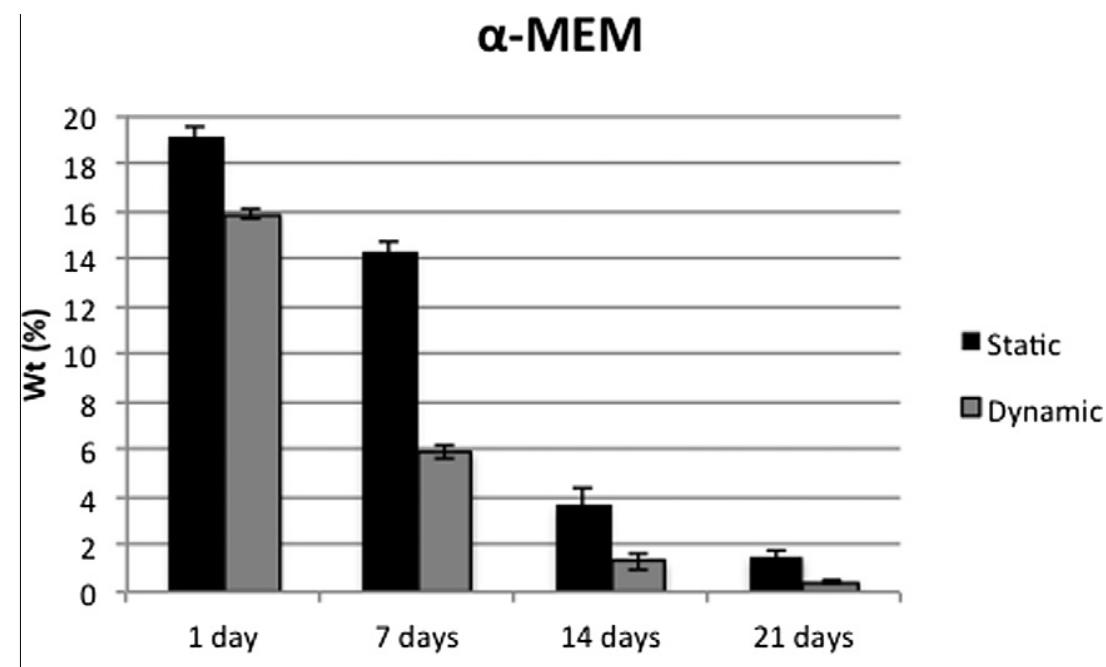

\section{Osteogenic medium}

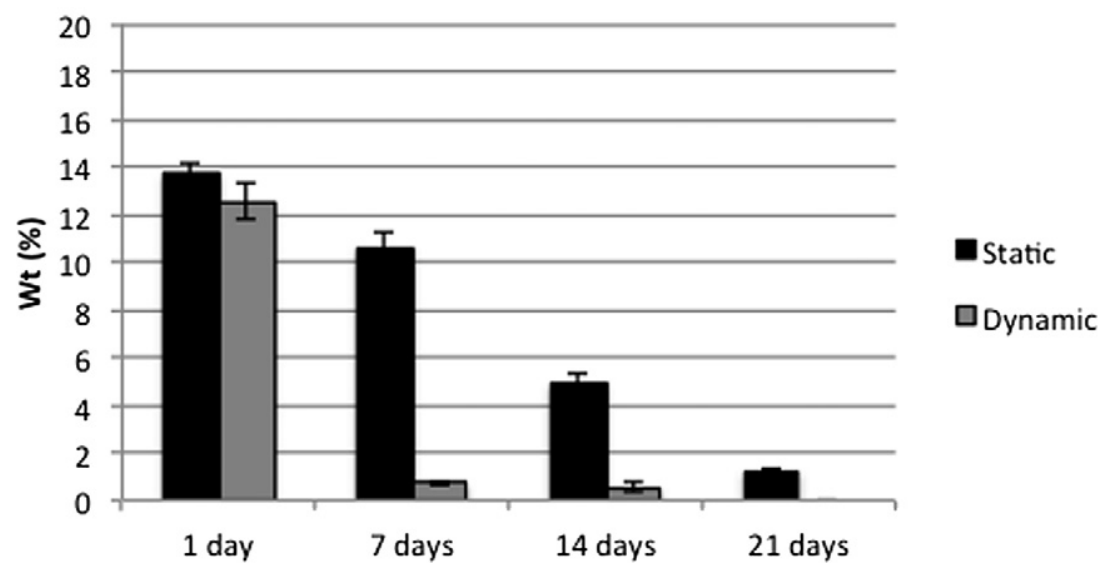

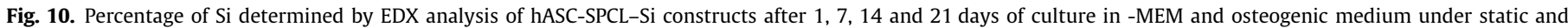
dynamic conditions. Data are shown as mean \pm standard deviation from $n=3$ samples.

For ALP activity in Fig. 6, it can be observed that at day 1 the activity was lower, indicating that the osteogenic differentiation of the cultured cells does not occur in the first $24 \mathrm{~h}$. These results are in agreement with the results from EDX analysis, since the $\mathrm{P}$ element was not detected on the surface of SPCL-Si after 1 day of culture. From day 1 to day 7, an increase in ALP activity was observed for both materials under all culture conditions. Thus, it could be an indication of the beginning of hASC differentiation. Also, the results showed a significantly $(p<0.05)$ higher activity under dynamic culture conditions in osteogenic medium compared with the static conditions in $\alpha$-MEM, for both SPCL and SPCL-Si fibre meshes. These results are supported by other studies with hASC [35]. Concerning the culture medium, some research works $[37,38]$ showed that the addition of osteogenic supplements to hASC induced rapid osteogenesis, detected by the increased expression of ALP. Also, it is believed [39] that perfusion culture provides better micro-environmental conditions for the stem cells to populate and differentiate into an osteogenic pathway. The observed increase in the ALP activity under dynamic conditions for SPCL and SPCL-Si scaffolds in both culture media can be related to the continuous and larger supply of culture media by the bioreactor, which allows a more rapid and efficient refresh of exhausted cellular metabolites during hASC mitosis and differentiation. At the same time, dynamic culture conditions provide mechanical stimulus to the cells via the shear stresses induced by the flow perfusion. However, in the case of SPCL-Si constructs under static and dynamic conditions, the ALP present lower values, which may be associated with the $\mathrm{Ca}$ and $\mathrm{Si}$ ions present in the coagulation bath, which may decrease the activity of ALP of the cells, thus delaying the differentiation process. This delay, however, does not compromise the full osteogenic differentiation of the hASC, as demonstrated by the results showing the occurrence of mineralization after 14 days of culture. The functionalized surface of the SPCL-Si scaffolds using a calcium silicate solution can delay the osteogenic differentiation, owing to the presence of Ca ions, and lead to lower ALP expression, as ALP is an early osteogenic differentiation marker [40]. The same behaviour was observed in different studies [40,41], where the ALP expression was higher in untreated samples than in samples coated with a calcium phosphate layer. Also, another work [42] reported that calcium negatively regulates the net release of ALP activity when using human osteosarcoma ( $\mathrm{SaOs}-2$ ) cells. As ALP analysis is a controversial technique, mainly in bioactive materials, future experiments are needed to understand better the effect of the presence of $\mathrm{Si}$ and $\mathrm{Ca}$ ions on the ALP expression. Besides that, there are several variations between in vitro and in vivo microenvironments that are mainly due to the difference between 
the dynamic of body fluids in vivo and the static microenviroment in vitro.

FTIR-ATR analysis on cell-seeded constructs revealed some differences between SPCL and SPCL-Si fibre meshes (Fig. 7). The presence of amide bands in both constructs for all the conditions can be related to the protein matrix formed. The most prevalent change observed was the presence of a well-defined phosphate band at $1078 \mathrm{~cm}^{-1}$ on SPCL-Si scaffolds cultured with cells for all conditions, especially in the case of $\alpha$-MEM (Fig. 7 , condition 2). This is indicative of the formation of a mineralized matrix due to the osteogenic differentiation of hASC promoted by the presence of $\mathrm{Si}-\mathrm{OH}$ groups. In the case of SPCL scaffolds, a phosphate band was also detected at $\sim 1090 \mathrm{~cm}^{-1}$, although the intensity is significantly lower than for SPCL-Si materials (Fig. 7). Also, this band is difficult to identify because of overlapping of bands of starch, i.e., the bands related to $-\mathrm{C}-\mathrm{O}-\mathrm{C}-$ of glycosidic bonds typically from starch. The presence of this band in the SPCL materials can be related to the deposition of residual amounts of phosphorous present in both culture media, as it can be seen in the spectra that its intensity differs between the static (conditions 2 and 3 ) and dynamic conditions (conditions 4 and 5). This assumption is supported by SEM observation and EDX analysis (Fig. 8), where it was not possible to observe the formation of mineralization nodules in SPCL materials as well as to detect the presence of P.

For SPCL-Si scaffolds, the mineralization nodules were observed after 14 days under osteogenic medium/static condition (Fig. 8). These results demonstrate clear differences in the formation of mineralization nodules between SPCL and SPCL-Si scaffolds. Once more, these results can be related to the possible release of Si ions to the medium (Fig. 10). The EDX spectra confirmed the presence of $\mathrm{P}$ and $\mathrm{Ca}$ elements on the mineralization nodules. In contrast, in the cultured scaffolds under dynamic conditions, the presence of mineralization nodules was not observed. However, the FTIR spectra (Fig. 7) and EDX spectra (Fig. 8) showed the presence of phosphate bands and $\mathrm{P}$ in those materials under dynamic conditions, which indicates that the mineralization occurred even though the presence of mineralization nodules was not observed. The non-existence of the mineralization nodules may be explained by the flow provided by the bioreactor, which does not allow high mineral depositions. For instance, Gough et al. [43] obtained similar results with $58 \mathrm{~S}$ bioactive glass scaffolds. EDX analysis of SPCL scaffolds revealed the non-existence of $\mathrm{P}$ and $\mathrm{Ca}$ ions on these materials related to the non-existence of mineralization nodules (Fig. 8).

From all these data, one can speculate that after 14 days of culture in osteogenic medium, the cells cultured on SPCL-Si scaffolds are able to differentiate for producing a mineralized extracellular matrix.

In the first $24 \mathrm{~h}$ of culture, the presence of $\mathrm{P}$ on the surface of SPCL-Si was not detected, which indicates that osteogenic differentiation did not occur (Fig. 9). For SPCL-Si fibre meshes in $\alpha$ MEM after 14 and 21 days, in both static and dynamic conditions, the $\mathrm{Ca} / \mathrm{P}$ ratios are very close to those found for the hydroxyapatite present in natural bone (1.67) [44] (Fig. 9). Oh et al. [39] demonstrated that bioactive scaffolds cultured with hASC under dynamic conditions show similar results to those obtained in the present work. These results demonstrate that the supporting capacity of $\mathrm{Si}-\mathrm{OH}$ groups incorporated into the SPCL scaffolds can promote differentiation of hASC into osteoblasts and produce a mineralized matrix. Moreover, these results are in agreement with the percentage of Si determined by EDX analysis (Fig. 10). A decrease in Si with the culturing time, in either $\alpha$-MEM or osteogenic medium, was observed, which indicates that it is being released into the culture medium and therefore influences the differentiation of the cells (Fig. 10).
Thus, according to the present results, the presence of osteogenic factors in the culture medium may not be strictly necessary to obtain osteogenic differentiation of hASC. Also, this is in agreement with the detection of phosphate bands in the FTIR spectra for scaffolds cultured in $\alpha$-MEM (Fig. 7, condition 2 ). The $\mathrm{Ca} / \mathrm{P}$ ratios in SPCL-Si scaffolds reached higher values in osteogenic medium under dynamic culture, which is related to the presence of osteogenic factors in the culture medium and the flow rate used in the bioreactor, which plays an important role on cell proliferation and differentiation. Also, a possible explanation for this can be the affinity of the $\mathrm{Si}-\mathrm{OH}$ to bind calcium ions. Once more, these results are in agreement with those obtained from the ALP activity (Fig. 6). Under dynamic culture in osteogenic medium, the values of the $\mathrm{Ca} / \mathrm{P}$ ratio and ALP activity are higher, indicating that the mineralization can occur mainly in the SPCL-Si scaffolds. For the SCPL-Si in $\alpha$-MEM, controls without cells, the presence of $P$ was not detected, which can be a positive indication that the $P$ detected in cell-cultured SPCL-Si constructs comes from mineralized matrix produced by hASC. However, since this work is considered a first step towards the development of highperformance materials for bone regeneration, more biological characterization is needed, namely, gene expression and histological analysis, for osteocalcin, osteopontin and collagen type I, to understand the influence of $\mathrm{Si}$ and $\mathrm{Ca}$ ions, either individually or combined, in osteogenic differentiation. Overall, the results demonstrated that polymeric wet-spun fibre meshes incorporating bioactive functional groups, $\mathrm{Si}-\mathrm{OH}$, has potential as a supporting matrix for hASC osteogenic differentiation. This methodology allowed the development of 3-D wide-net mesh by assembling these thin wet-spun fibre meshes, and allowed their structure to be adjusted according to the location of implantation and size of bone defect.

\section{Conclusions}

The present study has demonstrated the chemical and biological importance of the presence of silicon in bone TE strategies. The combination of wet-spinning technology and a calcium silicate solution allows a bioactive starch-based scaffold with $\mathrm{Si}-\mathrm{OH}$ functional groups to be developed. This simple methodology avoids the use of calcium phosphate coating or chemical modification of the fibre mesh to obtain a bioactive scaffold. Osteogenic development of the hASC under the presence of $\mathrm{Si}-\mathrm{OH}$ groups was considerably up-regulated by dynamic cell culture, flow perfusion, compared with static culture, which suggests that the use of these functionalized scaffolds may eliminate the need to use osteogenic supplemented medium. During 14 days of culture, SPCL-Si scaffolds sustained hASC proliferation and differentiation into the osteogenic lineage. These data suggest the potential of SPCL scaffolds functionalized with $\mathrm{Si}-\mathrm{OH}$ groups seeded with hASC and cultured under flow perfusion to be considered as a promising biomaterial for bone tissue replacement. Moreover, the favourable in vitro results presented here highlight the need to pursue further investigation on the effects that $\mathrm{Si}$ and $\mathrm{Ca}$ have on cellular response such as cell differentiation, cell migration and cell adhesion. The important of this investigation relies on the ability to engineer smart bioactive materials with tailored ion release kinetics and controlled biological response in the relevant physiological environment of bone tissue.

\section{Disclosures}

No competing financial interests exist. 


\section{Acknowledgements}

A.I. Rodrigues and I.B. Leonor thank the Portuguese Foundation for Science and Technology (FCT) for providing support: PhD scholarship (Grant No. SFRH/BD/69962/2010) and a post-doctoral scholarship (Grant No. SFRH/BPD/26648/2006). This work was supported by the European NoE EXPERTISSUES (NMP3-CT-2004500283) and by the Portuguese Foundation for Science and Technology (FCT), through projects PTDC/CTM/67560/2006.

\section{A. Figures with essential colour discrimination}

Certain figures in this article, particularly Figs. 1 and 2, are difficult to interpret in black and white. The full color images can be found in the on-line version, at http://dx.doi.org/10.1016/ j.actbio.2012.05.025.

\section{Appendix B. Supplementary data}

Supplementary data associated with this article can be found, in the online version, at http://dx.doi.org/10.1016/j.actbio.2012. 05.025.

\section{References}

[1] Puppi D, Chiellini F, Piras AM, Chiellini E. Polymeric materials for bone and cartilage repair. Prog Polymer Sci 2010;35(4):403-40.

[2] Marot D, Knezevic M, Novakovic GV. Bone tissue engineering with human stem cells. Stem Cell Res Ther 2010;1(2):1-10.

[3] Laurencin CT, Ambrosio AMA, Borden MD, Cooper JA. Tissue engineering: orthopedic applications. Annu Rev Biomed Eng 1999;01:19-46.

[4] Liu XH, Ma PX. Polymeric scaffolds for bone tissue engineering. Ann Biomed Eng 2004;32(3):477-86.

[5] Giannoudis PV, Dinopoulos H, Tsiridis E. Bone substitutes: an update. InjuryInternational. J Care Injured 2005;36(3):S20-7.

[6] Ni SY, Chang J, Chou L, Zhai WY. Comparison of osteoblast-like cell responses to calcium silicate and tricalcium phosphate ceramics in vitro. J Biomed Mater Res Part B-Appl Biomater 2007;80B(1):174-83.

[7] Shin K-H, Sung J-H, Koh Y-H, Lee J-H, Choi W-Y, Kim H-E. Direct coating of bioactive sol-gel derived silica on poly(epsilon-caprolactone) nanofibrous scaffold using co-electrospinning. Mater Lett 2010;64:1539-42.

[8] Nair MB, Varma H, Shenoy SJ, John A. Treatment of goat femur segmental defects with silica-coated hydroxyapatiteone-year follow-up. Tissue Eng Part A 2010;16(2):385-91.

[9] Gomes ME, Sikavitsas VI, Behravesh E, Reis RL, Mikos AG. Effect of flow perfusion on the osteogenic differentiation of bone marrow stromal cells cultured on starch-based three-dimensional scaffolds. J Biomed Mater Res, Part A 2003;67:87-95.

[10] Bancroft GN, Sikavitsas VI, Mikos AG. Design of a flow perfusion bioreactor system for bone tissue-engineering applications. Tissue Eng 2003;9(3): 549-54.

[11] Gomes ME, Reis RL, Mikos AG. Bone tissue engineering constructs based on starch scaffolds and bone marrow cells cultured in a flow perfusion bioreactor. Mater Sci Forum 2006;980:514-6.

[12] Stiehler M, Bünger C, Baatrup A, Lind M, Kassem M, Mygind T. Effect of dynamic 3-D culture on proliferation, distribution, and osteogenic differentiation of human mesenchymal stem cells. J Biomed Mater Res, Part A 2009;89A(1):96-107.

[13] Azevedo HS, Gama FM, Reis RL. In vitro assessment of the enzymatic degradation of several starch based biomaterials. Biomacromolecules 2003;4:1703-12.

[14] Pashkuleva I, Azevedo HS, Reis RL. Surface structural investigation of starchbased biomaterials. Macromol Biosci 2008;8(2):210-9.

[15] Oyane A, Kawashita M, Nakanishi K, Kokubo T, Minoda M, Miyamoto T, et al. Bonelike apatite formation on ethylene-vinyl alcohol copolymer modified with silane coupling agent and calcium silicate solutions. Biomaterials 2003;24: 1729-35.

[16] Leonor IB, Rodrigues MT, Gomes ME, Reis RL. In situ functionalization of wetspun fibre meshes for bone tissue engineering. J Tissue Eng Regen Med 2011;5(2):104-11.

[17] Rada T, Reis RL, Gomes ME. Novel method for the isolation of adipose stem cells (ASCs). J Tissue Eng Regen Med 2009;3(2):158-9.

[18] Costa PF, Martins A, Neves NM, Gomes ME, Reis RL. Multichamber bioreactor with bidirectional perfusion integrated in culture system for tissue engineering strategies. PT 1041552009.
19] Bancroft GN, Sikavitsast VI, van den Dolder J, Sheffield TL, Ambrose CG, Jansen $J A$, et al. Fluid flow increases mineralized matrix deposition in threedimensional perfusion culture of marrow stromal osteoblasts in a dosedependent manner. Proc Nat Acad Sci USA 2002;99(20):12600-5.

[20] Carlisle EM. Silicon - a requirement in bone-formation independent of Vitamin D1. Calcif Tissue Int 1981;33(1):27-34.

[21] Hench LL. Genetic design of bioactive glass. J Eur Ceram Soc 2009;29(7): 1257-65.

[22] Pietak AM, Reid JW, Stott MJ, Sayer M. Silicon substitution in the calcium phosphate bioceramics. Biomaterials 2007;28(28):4023-32.

[23] Hing KA, Revell PA, Smith N, Buckland T. Effect of silicon level on rate, quality and progression of bone healing within silicate-substituted porous hydroxyapatite scaffolds. Biomaterials 2006;27(29):5014-26.

[24] Elzein T, Nasser-Eddine M, Delaite C, Bistac S, Dumas P. FTIR study of polycaprolactone chain organization at interfaces. J Colloid Interface Sci 2004;273(2):381-7.

[25] Nie K, Zheng S, Lu F, Zhu Q. Inorganic-organic hybrids involving poly $(\varepsilon$ caprolactone) and silica network: hydrogen-bonding interactions and isothermal crystallization kinetics. J Polym Sci, Part B: Polym Phys 2005;43: 2594-603.

[26] Eglin D, Ali SAM, Perry CC. A statistical study of poly( $\varepsilon$-caprolactone) crystallinity in poly( $\varepsilon$-caprolactone)-silica sol-gel materials and their in vitro calcium phosphate-forming ability. Polym Int 2003;52(12):1807-19.

[27] Taddei P, Tinti A, Reggiani M, Fagnano C. In vitro mineralization of bioresorbablepoly( $\varepsilon$-caprolactone)/apatite composites for bone tissue engineering: a vibrational and thermal investigation. J Mol Struct 2005;744747:135-43

[28] Rezwan K, Chen QZ, Blaker JJ, Bocaccini AR. Biodegradable and bioactive porous polymer/inorganic composite scaffolds for bone tissue engineering. Biomaterials 2006:27:3413-31.

[29] Bocaccini AR, Blaker JJ, Maquet V, Day RM, Jérôme R. Preparation and characterization of poly(lactide-co-glycolide) (PLGA) and PLGA/Bioglass ${ }^{\circledR}$ composite tubular foam scaffolds for tissue engineering applications. Mater Sci Eng C 2005;25:23-31.

[30] Ciapetti G, Ambrosio L, Savarino L, Granchi D, Cenni E, Baldini N, et al Osteoblast growth and function in porous poly epsilon-caprolactone matrices for bone repair: a preliminary study. Biomaterials 2003;24(21):3815-24.

[31] Haimi S, Suuriniemi N, Haaparanta A-M, Ellä V, Lindroos B, Huhtala H, et al. Growth and osteogenic differentiation of adipose stem cells on PLA/bioactive glass and PLA/beta-TCP scaffolds. Tissue Eng Part A 2009;15:1473-80.

[32] Zou S, Ireland D, Brooks RA, Rushton N, Best S. Effects of silicate ions on human osteoblast adhesion, proliferation, and differentiation. J Biomed Mater Res B Appl Biomater 2008;90(1):123-30.

[33] Gibson IR, Huang J, Best SM, Bonfield W. Enhanced in vitro cell activity and surface apatite layer formation on novel silicon substituted hydroxyapatites. In: Ohgushi $\mathrm{H}$, Hastings GW, Yoshikawa $\mathrm{T}$, editors. Proceedings of the International Symposium on Ceramics in Medicine, Bioceramics 12. River Edge, NJ: World Scientific Publishing; 1999. p. 191-4.

[34] Lin TM, Tsai JL, Lin SD, Lai CS, Chang CC. Accelerated growth and prolonged lifespan of adipose tissue-derived human mesenchymal stem cells in a medium using reduced calcium and antioxidants. Stem Cells Develop 2005; 14(1):92-102.

[35] Fröhlich M, Grayson WL, Marolt D, Gimble JM, Kregar-Velikonja N, VunjakNovakovic G. Bone grafts engineered from human adipose-derived stem cells in perfusion bioreactor culture. Tissue Eng Part A 2010;16(1):179-89.

[36] Aubin JE. Regulation of osteoblast formation and function. Rev Endocr \& Metab Disord 2001;2(81):81-94.

[37] Hattori H, Sato M, Masuoka K, Ishihara M, Kikuchi T, Matsui T, et al. Osteogenic potential of human adipose tissue-derived stromal cells as an alternative stem cell source. Cells Tissues Organs 2004;178(1):2-12.

[38] Hattori H, Masuoka K, Sato M, Ishihara M, Asazuma T, Takase B, et al. Bone formation using human adipose tissue-derived stromal cells and a biodegradable scaffold. J Biomed Mater Res Part B-Appl Biomater 2006;76(1):230-9.

[39] Oh CH, Hong S, Jeong I, Yu H, Jegal S, Kim H. Development of robotic dispensed bioactive scaffolds and human adipose-derived stem cell culturing for bone tissue engineering. Tissue Eng Part C-Methods 2010;16(4):561-71.

[40] Nandakumar A, Yang L, Habibovic P, van Blitterswijk C. Calcium phosphate coated electrospun fibre matrices as scaffolds for bone tissue engineering. Langmuir 2010;26(10):7380-7.

[41] Haimi S, Moimas L, Pirhonen E, Lindroos B, Huhtala H, Räty S, et al. Calcium phosphate surface treatment of bioactive glass causes a delay in early osteogenic differentiation of adipose stem cells. J Biomed Mater Res Part A 2009;91(2):540-7.

[42] Anh DJ, Dimai HP, Hall SL, Farley JR. Skeletal alkaline phosphatase activity is primarily released from human osteoblasts in an insoluble form, and the net release is inhibited by calcium and skeletal growth factors. Calcif Tissue Int 1998;62(4):332-40.

[43] Gough JE, Jones JR, Hench LL. Nodule formation and mineralisation of human primary osteoblasts cultured on a porous bioactive glass scaffold. Biomaterials 2004;25:2039-46.

[44] Palmer LC, Newcomb C, Kaltz SR, Spoerke ED, Stupp SI. Biomimetic systems for hydroxyapatite mineralization inspired by bone and enamel. Chem Rev 2008;108(11):4754-83. 